\title{
Improved Liu-Type Estimator of Parameters in Two Seemingly Unrelated Regressions
}

\author{
Jibo $W \mathbf{u}^{1,2}$ \\ ${ }^{1}$ School of Mathematics and Finances, Chongqing University of Arts and Sciences, Chongqing 402160, China \\ ${ }^{2}$ Department of Mathematics and KLDAIP, Chongqing University of Arts and Sciences, Chongqing 402160, China
}

Correspondence should be addressed to Jibo Wu; linfen52@126.com

Received 27 December 2013; Accepted 18 February 2014; Published 16 March 2014

Academic Editors: Y. M. Cheng and F. Sartoretto

Copyright (c) 2014 Jibo Wu. This is an open access article distributed under the Creative Commons Attribution License, which permits unrestricted use, distribution, and reproduction in any medium, provided the original work is properly cited.

We consider the parameter estimation in two seemingly unrelated regression systems. To overcome the multicollinearity, we propose a Liu-type estimator in seemingly unrelated regression systems. The superiority of the new estimator over the classic estimator in the mean square error is discussed and we also discuss the admissibility of the Liu-type estimator.

\section{Introduction}

Consider the following two seemingly unrelated regressions (SUR):

$$
\begin{aligned}
& Y_{1}=X_{1} \beta_{1}+\varepsilon_{1}, \\
& Y_{2}=X_{2} \beta_{2}+\varepsilon_{2},
\end{aligned}
$$

where $Y_{i}(i=1,2)$ are $n \times 1$ vectors of observations, $X_{i}(i=$ $1,2)$ are $n \times p$ matrices with full column rank, $\beta_{i}(i=1,2)$ are $p \times 1$ vectors of unknown regression parameters, $\varepsilon_{i}(i=1,2)$ are $n \times 1$ vectors of error variables, and

$$
E\left(\varepsilon_{i}\right)=0, \quad \operatorname{Cov}\left(\varepsilon_{i}, \varepsilon_{j}\right)=\sigma_{i j} I_{n}, \quad i, j=1,2,
$$

where $V=\left(\sigma_{i j}\right)$ is a $2 \times 2$ positive definite matrix. This system has been used in many fields, such as social biological sciences and econometrics. Zellner $[1,2]$ firstly defined this system and later it was discussed by many researchers, such as Wang et al. [3], Roozbeh et al. [4], and Singh et al. [5].

For system (1), when $\sigma_{12}=0$, we can get the estimator of $\beta$ as follows:

$$
\widehat{\beta}=\left(\begin{array}{l}
\widehat{\beta}_{1} \\
\widehat{\beta}_{2}
\end{array}\right)=\left(\begin{array}{l}
\left(X_{1}^{\prime} X_{1}\right)^{-1} X_{1}^{\prime} Y_{1} \\
\left(X_{2}^{\prime} X_{2}\right)^{-1} X_{2}^{\prime} Y_{2}
\end{array}\right) .
$$

When the error vector is related, the estimator $\widehat{\beta}$ may not fully use the information of the parameter. How to use the information to improve the $\widehat{\beta}$ is a problem. Revankar [6] and Srivastava and Giles [7] have discussed this problem. Wang [8] uses the covariance-improve method to estimate the parameter in two seemingly unrelated regressions. With the prior information, Wang [8] proposes the covarianceimprove estimator of $\beta_{1}$ and $\beta_{2}$ :

$$
\begin{aligned}
& \tilde{\beta}_{1}=\left(X_{1}^{\prime} X_{1}\right)^{-1} X_{1}^{\prime} Y_{1}-\frac{\sigma_{12}}{\sigma_{22}}\left(X_{1}^{\prime} X_{1}\right)^{-1} X_{1}^{\prime} N_{2} Y_{2}, \\
& \tilde{\beta}_{2}=\left(X_{2}^{\prime} X_{2}\right)^{-1} X_{2}^{\prime} Y_{2}-\frac{\sigma_{12}}{\sigma_{11}}\left(X_{2}^{\prime} X_{2}\right)^{-1} X_{2}^{\prime} N_{1} Y_{1},
\end{aligned}
$$

where $N_{j}=I-P_{j}, P_{j}=X_{j}\left(X_{j} X_{j}^{\prime}\right)^{-1} X_{j}^{\prime}, j=1,2$.

When the design matrix $X_{i}$ is ill-conditioned, the estimator $\widetilde{\beta}_{i}, i=1,2$ is no longer a good estimator. Many researchers have discussed this problem. One method to overcome this problem is to consider biased estimator, such as Liu and Wang [9] introduce the ridge estimator, Liu [10] proposes the principal regression component estimator, Qiu [11] proposes the classic $c-k$ type estimator, and Roozbeh et al. [12] introduce the ridge estimator.

In this paper, we propose a Liu-type estimator to overcome the multicollinearity in the two seemingly unrelated regressions. Then we discuss the superiority of the Liu-type 
estimator over the covariance-improve estimator in the mean square error criterion, and we also discuss the selection of the parameter in the proposed estimator. Since the estimator of $\beta_{2}$ is similar to the estimator of $\beta_{1}$, so in this paper, we only discuss the estimator of $\beta_{1}$.

The rest of the paper is organized as follows. The Liu-type estimator is given in Section 2 and the properties of the new estimator under the mean square error criterion are discussed in Section 3. The admissibility of the proposed estimator is studied in Section 4 and some conclusion remarks are given in Section 5.

\section{The Proposed Estimator}

In this section, we use the prior information to obtain the Liutype estimator. Firstly, we give a lemma.
Lemma 1 (see [10]). Let $\widetilde{\theta}$ be an estimator of $\theta, \widehat{\theta}$ the prior information with $E(\widehat{\theta})=0$, and

$$
\operatorname{Cov}\left(\begin{array}{l}
\widetilde{\theta} \\
\widehat{\theta}
\end{array}\right)=\left(\begin{array}{ll}
V_{11} & V_{12} \\
V_{12} & V_{22}
\end{array}\right)
$$

Then in the estimator $A=\left\{\theta^{*}(X)=\widetilde{\theta}+X \widehat{\theta}\right\}$, the estimator $\theta^{*}=\widetilde{\theta}-V_{12} V_{22}^{-1} \widehat{\theta}$ has minimum mean square error and

$$
\operatorname{MSE}\left(\theta^{*}\right)=\operatorname{MSE}(\widetilde{\theta})-\operatorname{tr}\left(V_{12} V_{22}^{-1} V_{21}\right)
$$

Now we establish the Liu-type estimator in SUR model. Set $Z_{2}$ satisfy $X_{2}^{\prime} Z_{2}=0$ and $X_{2}$ has the max column full rank; then $E\left(Z_{2}^{\prime} Y_{2}\right)=0$ and

$$
\operatorname{Cov}\left(\begin{array}{c}
\widehat{\beta}_{1}(k, d) \\
Z_{2}^{\prime} Y_{2}
\end{array}\right)=\left(\begin{array}{cc}
\sigma_{11}\left(X_{1}^{\prime} X_{1}+k I\right)^{-2}\left(X_{1}^{\prime} X_{1}+d I\right)^{2}\left(X_{1}^{\prime} X_{1}\right)^{-1} & \sigma_{12}\left(X_{1}^{\prime} X_{1}+k I\right)^{-1}\left(X^{\prime} X+d I\right)\left(X_{1}^{\prime} X_{1}\right)^{-1} X_{1}^{\prime} Z_{2} \\
\sigma_{12} Z_{2}^{\prime} X_{1}\left(X_{1}^{\prime} X_{1}\right)^{-1}\left(X_{1}^{\prime} X_{1}+k I\right)^{-1}\left(X^{\prime} X+d I\right) & \sigma_{22} Z_{2}^{\prime} Z_{2}
\end{array}\right)
$$

By Lemma 1, when $V$ is known, we establish a new estimator for $\beta_{1}$ :

$$
\begin{aligned}
\widetilde{\beta}_{1}(k, d)= & \widehat{\beta}_{1}(k, d)-\frac{\sigma_{12}}{\sigma_{22}}\left(X_{1}^{\prime} X_{1}+k I\right)^{-1} \\
& \times\left(X_{1}^{\prime} X_{1}+d I\right)\left(X_{1}^{\prime} X_{1}\right)^{-1} X_{1}^{\prime} Z_{2}\left(Z_{2}^{\prime} Z_{2}\right)^{-1} Z_{2}^{\prime} Y_{2} \\
= & \left(X_{1}^{\prime} X_{1}+k I\right)^{-1}\left(X_{1}^{\prime} X_{1}+d I\right)\left(X_{1}^{\prime} X_{1}\right)^{-1} X_{1}^{\prime} Y_{1} \\
& -\frac{\sigma_{12}}{\sigma_{22}}\left(X_{1}^{\prime} X_{1}+k I\right)^{-1}\left(X_{1}^{\prime} X_{1}+d I\right) \\
& \times\left(X_{1}^{\prime} X_{1}\right)^{-1} X_{1}^{\prime} N_{2} Y_{2},
\end{aligned}
$$

where $\widehat{\beta}_{1}(k, d)=\left(X_{1}^{\prime} X_{1}+k I\right)^{-1}\left(X^{\prime} X+d I\right)\left(X_{1}^{\prime} X_{1}\right)^{-1} X_{1}^{\prime} Y_{1}$ is the estimator for the single model in (1), $k>0,0<d<1, d<$ $k, N_{2}=I-P_{2}$, and $P_{2}=X_{2}\left(X_{2}^{\prime} X_{2}\right)^{-1} X_{2}$. We call this estimator as Liu-type estimator in SUR model. By the definition of the Liu-type estimator, it is easy to know that it is a general estimator which includes the covariance-improve estimator proposed by Wang [8] and the ridge estimator proposed by Liu [10] as special cases.

(1) When $k=d=0$, then

$$
\tilde{\beta}_{1}(k, d)=\tilde{\beta}_{1}=\left(X_{1}^{\prime} X_{1}\right)^{-1} X_{1}^{\prime} Y_{1}-\frac{\sigma_{12}}{\sigma_{22}}\left(X_{1}^{\prime} X_{1}\right)^{-1} X_{1}^{\prime} N_{2} Y_{2} .
$$

(2) When $d=1$, then

$$
\begin{aligned}
\widetilde{\beta}_{1}(k, d)= & \widetilde{\beta}_{1}(k) \\
= & \left(X_{1}^{\prime} X_{1}+k I\right)^{-1} X_{1}^{\prime} Y_{1} \\
& -\frac{\sigma_{12}}{\sigma_{22}}\left(X_{1}^{\prime} X_{1}+k I\right)^{-1} X_{1}^{\prime} N_{2} Y_{2} .
\end{aligned}
$$

In next section, we will give the properties of the new estimator.

\section{MSE: The Superiority of the New Estimator over the Covariance-Improve Estimator}

In this section, we discuss the statistical properties of the new estimator $\widetilde{\beta}(k, d)$. Firstly, we give the definition of the mean square error (MSE).

Let $b^{*}$ be an estimator of $\beta$; the MSE of $b^{*}$ is defined as

$$
\begin{aligned}
\operatorname{MSE}\left(b^{*}, \beta\right) & =E\left[\left(b^{*}-\beta\right)^{\prime}\left(b^{*}-\beta\right)\right] \\
& =\operatorname{tr}\left(\operatorname{Cov}\left(b^{*}\right)\right)+\left(\operatorname{bias}\left(b^{*}\right)\right)^{\prime}\left(\operatorname{bias}\left(b^{*}\right)\right),
\end{aligned}
$$

where $\operatorname{bias}\left(b^{*}\right)=E\left(b^{*}\right)-\beta$.

In the following theorem, we give the superiority of the new estimator over the covariance-improve estimator in the mean square error.

Theorem 2. For the SUR model (1), when $V$ is known,

(a) when $0<d<1$ fixed,

(1) if $\sigma_{11}\left(1 / \lambda_{i}-\rho_{12}^{2}\left(a_{i i} / \lambda_{i}^{2}\right)\right)-\alpha_{i}^{2} \geq 0$, then $\operatorname{MSE}\left(\widetilde{\beta}_{1}\right) \geq \operatorname{MSE}\left(\widetilde{\beta}_{1}(k, d)\right)$;

(2) if $\sigma_{11}\left(1 / \lambda_{i}-\rho_{12}^{2}\left(a_{i i} / \lambda_{i}^{2}\right)\right)-\alpha_{i}^{2}<0$, when $0<k<$ $\left(\sigma_{11}\left(2 \lambda_{i}+d\right)\left(1 / \lambda_{i}+\rho_{12}^{2}\left(a_{i i} / \lambda_{i}^{2}\right)\right)+d \alpha_{i}^{2}\right) /\left(\alpha_{i}^{2}-\right.$ $\left.\sigma_{11}\left(1 / \lambda_{i}-\rho_{12}^{2}\left(a_{i i} / \lambda_{i}^{2}\right)\right)\right)$, then $\operatorname{MSE}\left(\widetilde{\beta}_{1}\right) \geq$ $\operatorname{MSE}\left(\widetilde{\beta}_{1}(k, d)\right)$;

(b) when $k>0$ fixed,

(1) if $\sigma_{11}\left(2 \lambda_{i}+k\right)\left(1 / \lambda_{i}+\rho_{12}^{2}\left(a_{i i} / \lambda_{i}^{2}\right)\right) \geq k \alpha_{i}^{2}$, then $\operatorname{MSE}\left(\widetilde{\beta}_{1}\right) \geq \operatorname{MSE}\left(\widetilde{\beta}_{1}(k, d)\right)$; 
(2) if $\sigma_{11}\left(2 \lambda_{i}+k\right)\left(1 / \lambda_{i}+\rho_{12}^{2}\left(a_{i i} / \lambda_{i}^{2}\right)\right)<k \alpha_{i}^{2}$, when $\left(k \alpha_{i}^{2}-\sigma_{11}\left(2 \lambda_{i}+k\right)\left(1 / \lambda_{i}+\rho_{12}^{2}\left(a_{i i} / \lambda_{i}^{2}\right)\right)\right) /$ $\left(\sigma_{11}\left(1 / \lambda_{i}-\rho_{12}^{2}\left(a_{i i} / \lambda_{i}^{2}\right)\right)+\alpha_{i}^{2}\right) \leq d<1$, then $\operatorname{MSE}\left(\widetilde{\beta}_{1}\right) \geq \operatorname{MSE}\left(\widetilde{\beta}_{1}(k, d)\right)$.

Proof. By the definition of the covariance-improve estimator $\widetilde{\beta}_{1}$, we obtain

$$
\begin{aligned}
& E\left(\widetilde{\beta}_{1}\right)=\beta_{1}, \\
& \operatorname{Cov}\left(\widetilde{\beta}_{1}\right) \\
& =\sigma_{11}\left(X_{1}^{\prime} X_{1}\right)^{-1}-\frac{\sigma_{12}^{2}}{\sigma_{22}}\left(X_{1}^{\prime} X_{1}\right)^{-1} X_{1}^{\prime} N_{2} X_{1}\left(X_{1}^{\prime} X_{1}\right)^{-1} .
\end{aligned}
$$

Then by the definition of the mean square error, we obtain

$$
\begin{aligned}
\operatorname{MSE}\left(\widetilde{\beta}_{1}\right)= & \operatorname{tr}\left(\operatorname{Cov}\left(\widetilde{\beta}_{1}\right)\right)=\sigma_{11} \operatorname{tr}\left(\left(X_{1}^{\prime} X_{1}\right)^{-1}\right) \\
& -\frac{\sigma_{12}^{2}}{\sigma_{22}} \operatorname{tr}\left(\left(X_{1}^{\prime} X_{1}\right)^{-1} X_{1}^{\prime} N_{2} X_{1}\left(X_{1}^{\prime} X_{1}\right)^{-1}\right) .
\end{aligned}
$$

Since $X_{1}^{\prime} X_{1}>0$, there exists an orthogonal matrix such that $Q^{\prime} X_{1}^{\prime} X_{1} Q=\Lambda=\operatorname{diag}\left(\lambda_{1}, \ldots, \lambda_{p 1}\right)$, where $\lambda_{1} \geq \cdots \geq \lambda_{p 1}$ is the ordered eigenvalues of $X_{1}^{\prime} X_{1}$; thus we obtain

$$
\operatorname{MSE}\left(\widetilde{\beta}_{1}\right)=\sigma_{11} \sum_{i=1}^{p 1}\left(\frac{1}{\lambda_{i}}-\rho_{12}^{2} \frac{a_{i i}}{\lambda_{i}^{2}}\right)
$$

where $A=Q^{\prime} X_{1}^{\prime} N_{2} X_{1} Q=\left(a_{i j}\right)$. Since $\operatorname{Cov}\left(\widetilde{\beta}_{1}\right) \geq 0$ so we get that $1 / \lambda_{i}-\rho_{12}^{2}\left(a_{i i} / \lambda_{i}^{2}\right) \geq 0, i=1, \ldots, p 1$.

Now we compute the MSE of the Liu-type estimator $\left(\widetilde{\beta}_{1}(k, d)\right)$ for SUR model (1). Firstly, we compute that

$$
\begin{aligned}
E\left(\widetilde{\beta}_{1}(k, d)\right) & =\left(X_{1}^{\prime} X_{1}+k I\right)^{-1}\left(X_{1}^{\prime} X_{1}+d I\right) \beta_{1}, \\
\operatorname{Cov}\left(\widetilde{\beta}_{1}(k, d)\right)= & \left(X_{1}^{\prime} X_{1}+k I\right)^{-1}\left(X_{1}^{\prime} X_{1}+d I\right) \\
& \times \operatorname{Cov}\left(\widetilde{\beta}_{1}\right)\left(X_{1}^{\prime} X_{1}+d I\right)\left(X_{1}^{\prime} X_{1}+k I\right)^{-1} .
\end{aligned}
$$

Thus we have

$$
\begin{aligned}
\operatorname{MSE}\left(\widetilde{\beta}_{1}(k, d)\right)= & \operatorname{tr}\left(\operatorname{Cov}\left(\widetilde{\beta}_{1}(k, d)\right)\right) \\
& +\operatorname{bias}\left(\widetilde{\beta}_{1}(k, d)\right)^{\prime} \operatorname{bias}\left(\widetilde{\beta}_{1}(k, d)\right)^{\prime} \\
= & \sigma_{11} \sum_{i=1}^{p 1} \frac{\left(\lambda_{i}+d\right)^{2}}{\left(\lambda_{i}+k\right)^{2}}\left(\frac{1}{\lambda_{i}}-\rho_{12}^{2} \frac{a_{i i}}{\lambda_{i}^{2}}\right) \\
& +(k-d)^{2} \sum_{i=1}^{p 1} \frac{\alpha_{i}^{2}}{\left(\lambda_{i}+k\right)^{2}},
\end{aligned}
$$

In order to compare $\widetilde{\beta}_{1}(k, d)$ and $\widetilde{\beta}_{1}$, we consider the following difference:

$$
\begin{aligned}
& \operatorname{MSE}\left(\widetilde{\beta}_{1}\right)-\operatorname{MSE}\left(\widetilde{\beta}_{1}(k, d)\right) \\
& =\sigma_{11} \sum_{i=1}^{p 1}\left(\frac{1}{\lambda_{i}}-\rho_{12}^{2} \frac{a_{i i}}{\lambda_{i}^{2}}\right)-\sigma_{11} \sum_{i=1}^{p 1} \frac{\left(\lambda_{i}+d\right)^{2}}{\left(\lambda_{i}+k\right)^{2}}\left(\frac{1}{\lambda_{i}}-\rho_{12}^{2} \frac{a_{i i}}{\lambda_{i}^{2}}\right) \\
& -(k-d)^{2} \sum_{i=1}^{p 1} \frac{\alpha_{i}^{2}}{\left(\lambda_{i}+k\right)^{2}} \\
& =\sum_{i=1}^{p 1} \frac{(k-d)}{\left(\lambda_{i}+k\right)^{2}}\left[\left(2 \lambda_{i}+k+d\right)\left(\frac{1}{\lambda_{i}}-\rho_{12}^{2} \frac{a_{i i}}{\lambda_{i}^{2}}\right)-(k-d) \alpha_{i}^{2}\right] .
\end{aligned}
$$

When $0<d<1$ fixed, then we write (17) as follows:

$$
\begin{gathered}
\operatorname{MSE}\left(\widetilde{\beta}_{1}\right)-\operatorname{MSE}\left(\widetilde{\beta}_{1}(k, d)\right) \\
=\sum_{i=1}^{p 1} \frac{(k-d)}{\left(\lambda_{i}+k\right)^{2}}\left\{\left[\sigma_{11}\left(2 \lambda_{i}+d\right)\left(\frac{1}{\lambda_{i}}+\rho_{12}^{2} \frac{a_{i i}}{\lambda_{i}^{2}}\right)+d \alpha_{i}^{2}\right]\right. \\
\left.+k\left[\sigma_{11}\left(\frac{1}{\lambda_{i}}-\rho_{12}^{2} \frac{a_{i i}}{\lambda_{i}^{2}}\right)-\alpha_{i}^{2}\right]\right\} .
\end{gathered}
$$

So

(1) If $\sigma_{11}\left(1 / \lambda_{i}-\rho_{12}^{2}\left(a_{i i} / \lambda_{i}^{2}\right)\right)-\alpha_{i}^{2} \geq 0$, then $\operatorname{MSE}\left(\widetilde{\beta}_{1}\right) \geq$ $\operatorname{MSE}\left(\widetilde{\beta}_{1}(k, d)\right)$;

(2) if $\sigma_{11}\left(1 / \lambda_{i}-\rho_{12}^{2}\left(a_{i i} / \lambda_{i}^{2}\right)\right)-\alpha_{i}^{2}<0$, when $0<k<$ $\left(\sigma_{11}\left(2 \lambda_{i}+d\right)\left(1 / \lambda_{i}+\rho_{12}^{2}\left(a_{i i} / \lambda_{i}^{2}\right)\right)+d \alpha_{i}^{2}\right) /\left(\alpha_{i}^{2}-\sigma_{11}\left(1 / \lambda_{i}-\right.\right.$ $\left.\left.\rho_{12}^{2}\left(a_{i i} / \lambda_{i}^{2}\right)\right)\right)$, then $\operatorname{MSE}\left(\widetilde{\beta}_{1}\right) \geq \operatorname{MSE}\left(\widetilde{\beta}_{1}(k, d)\right)$.

When $k>0$ fixed, then we write (17) as follows:

$$
\begin{gathered}
\operatorname{MSE}\left(\widetilde{\beta}_{1}\right)-\operatorname{MSE}\left(\widetilde{\beta}_{1}(k, d)\right) \\
=\sum_{i=1}^{p 1} \frac{(k-d)}{\left(\lambda_{i}+k\right)^{2}}\left\{\left[\sigma_{11}\left(2 \lambda_{i}+k\right)\left(\frac{1}{\lambda_{i}}+\rho_{12}^{2} \frac{a_{i i}}{\lambda_{i}^{2}}\right)-k \alpha_{i}^{2}\right]\right. \\
\left.+d\left[\sigma_{11}\left(\frac{1}{\lambda_{i}}-\rho_{12}^{2} \frac{a_{i i}}{\lambda_{i}^{2}}\right)+\alpha_{i}^{2}\right]\right\} .
\end{gathered}
$$

So if

(1) $\sigma_{11}\left(2 \lambda_{i}+k\right)\left(1 / \lambda_{i}+\rho_{12}^{2}\left(a_{i i} / \lambda_{i}^{2}\right)\right) \geq k \alpha_{i}^{2}$, then $\operatorname{MSE}\left(\widetilde{\beta}_{1}\right) \geq \operatorname{MSE}\left(\widetilde{\beta}_{1}(k, d)\right)$;

(2) $\sigma_{11}\left(2 \lambda_{i}+k\right)\left(1 / \lambda_{i}+\rho_{12}^{2}\left(a_{i i} / \lambda_{i}^{2}\right)\right)<k \alpha_{i}^{2}$, when $\left(k \alpha_{i}^{2}-\sigma_{11}\left(2 \lambda_{i}+k\right)\left(1 / \lambda_{i}+\rho_{12}^{2}\left(a_{i i} / \lambda_{i}^{2}\right)\right)\right) /\left(\sigma_{11}\left(1 / \lambda_{i}-\right.\right.$ $\left.\left.\rho_{12}^{2}\left(a_{i i} / \lambda_{i}^{2}\right)\right)+\alpha_{i}^{2}\right) \leq d<1$, then $\operatorname{MSE}\left(\widetilde{\beta}_{1}\right) \geq$ $\operatorname{MSE}\left(\widetilde{\beta}_{1}(k, d)\right)$.

where $\alpha=Q^{\prime} \beta_{1}$. 


\section{The Admissible of the New Estimator $\widetilde{\beta}_{1}(k, d)$}

As we all know, the admissible of an estimator is an important problem in SUR model. In this section, we discuss the admissible of the new estimator $\widetilde{\beta}_{1}(k, d)$. Firstly, we give the definition of the admissible.

Definition 3. Let $\widehat{\theta}_{1}$ and $\widehat{\theta}_{2}$ be two estimators of $\theta$, for arbitrarily risk loss function $R(\cdot, \cdot)$, (1) $R\left(\widehat{\theta}_{1}, \theta\right) \leq R\left(\widehat{\theta}_{2}, \theta\right)$ for arbitrarily $\theta$. (2) There at least exist a $\theta_{0}$ such that the inequality is satisfied, Then we see that $\widehat{\theta}_{1}$ is uniformly better than $\widehat{\theta}_{2}$ for risk loss function $R(\cdot, \cdot)$. If, in some class estimators, there is not exist an estimator that is uniformly better than $\widetilde{\theta}$, we can say that $\widetilde{\theta}$ is admissible estimator of $\theta$ for risk loss function $R(\cdot, \cdot)$ in that class estimators.

Lemma 4 (see [13]). For linear model

$$
y=X \beta+e, \quad E(e)=0, \quad \operatorname{Cov}(e)=\sigma^{2} \Sigma,
$$

where $\Sigma>0$ is a known matrix and $X$ is full column rank. Let $T=X^{\prime} \Sigma^{-1} X, \beta^{*}(\Sigma)=T^{-1} X^{\prime} \Sigma^{-1} y$; then in the linear estimators of $S \beta, L \beta^{*}(\Sigma)$ is admissible estimator of $S \beta$ if and only if

$$
L T^{-1} L^{\prime} \leq L T^{-1} S^{\prime}
$$

Now we discuss the admissibility of the new estimator. In this section we consider the quadratic loss (mean square error).

Theorem 5. For the SUR model (1), when $V$ is know, if $X_{1}^{\prime} X_{2}=0$, then $\widetilde{\beta}_{1}(k, d)$ is admissible estimator of $\beta_{1}$ in the class of linear estimators $\Phi$.

Proof. If $X_{1}^{\prime} X_{2}=0$, then

$$
\begin{aligned}
\tilde{\beta}_{1}(k, d)= & \left(X_{1}^{\prime} X_{1}+k I\right)^{-1}\left(X_{1}^{\prime} X_{1}+d I\right)\left(X_{1}^{\prime} X_{1}\right)^{-1} X_{1}^{\prime} Y_{1} \\
- & \frac{\sigma_{12}}{\sigma_{22}}\left(X_{1}^{\prime} X_{1}+k I\right)^{-1} \\
& \times\left(X_{1}^{\prime} X_{1}+d I\right)\left(X_{1}^{\prime} X_{1}\right)^{-1} X_{1}^{\prime} Y_{2} .
\end{aligned}
$$

For SUR model (1), let $Y=\left(Y_{1}^{\prime}, Y_{2}^{\prime}\right)^{\prime}, X=\operatorname{diag}\left(X_{1}, X_{2}\right), \beta=$ $\left(\beta_{1}^{\prime}, \beta_{2}^{\prime}\right)$, and $\varepsilon=\left(\varepsilon_{1}^{\prime}, \varepsilon_{2}^{\prime}\right)$, Then (1) can be written as

$$
Y=X \beta+\varepsilon, \quad E(\varepsilon)=0, \quad \operatorname{Cov}(\varepsilon)=V,
$$

where

$$
V=\left(\begin{array}{ll}
\sigma_{11} I & \sigma_{12} I \\
\sigma_{21} I & \sigma_{22} I
\end{array}\right), \quad \beta^{*}=\left(\beta_{1}^{*}(V), \beta_{2}^{*}(V)\right)
$$

and (24) satisfies the following equation:

$$
X^{\prime} V^{-1} X \beta^{*}=X^{\prime} V^{-1} Y \text {. }
$$

Note that

$$
V^{-1}=\left(\sigma_{11} \sigma_{22}-\sigma_{12}^{2}\right)^{-1}\left(\begin{array}{cc}
\sigma_{22} I & -\sigma_{21} I \\
-\sigma_{12} I & \sigma_{11} I
\end{array}\right)
$$

Then (25) can be written as follows:

$$
\begin{gathered}
\left(\begin{array}{cc}
\sigma_{22} X_{1}^{\prime} X_{1} & -\sigma_{21} X_{1}^{\prime} X_{2} \\
-\sigma_{12} X_{2}^{\prime} X_{1} & \sigma_{11} X_{2}^{\prime} X_{2}
\end{array}\right)\left(\begin{array}{l}
\beta_{1}^{\prime *}(V) \\
\beta_{2}^{\prime *}(V)
\end{array}\right) \\
=\left(\begin{array}{cc}
\sigma_{22} X_{1}^{\prime} & -\sigma_{21} X_{1}^{\prime} \\
-\sigma_{12} X_{2}^{\prime} & \sigma_{11} X_{2}^{\prime}
\end{array}\right)\left(\begin{array}{l}
Y_{1} \\
Y_{2}
\end{array}\right) .
\end{gathered}
$$

When $X_{1}^{\prime} X_{2}=0$, (25) can be written as follows:

$$
\begin{gathered}
\left(\begin{array}{cc}
\sigma_{22} X_{1}^{\prime} X_{1} & 0 \\
0 & \sigma_{11} X_{2}^{\prime} X_{2}
\end{array}\right)\left(\begin{array}{l}
\beta_{1}^{\prime *}(V) \\
\beta_{2}^{\prime *}(V)
\end{array}\right) \\
=\left(\begin{array}{cc}
\sigma_{22} X_{1}^{\prime} & -\sigma_{21} X_{1}^{\prime} \\
-\sigma_{12} X_{2}^{\prime} & \sigma_{11} X_{2}^{\prime}
\end{array}\right)\left(\begin{array}{l}
Y_{1} \\
Y_{2}
\end{array}\right) .
\end{gathered}
$$

Then by (28), we may get

$$
X_{1}^{\prime} X_{1} \beta_{1}^{*}(V)=X_{1}^{\prime} Y_{1}-\frac{\sigma_{12}}{\sigma_{22}} X_{1}^{\prime} Y_{2}
$$

multiplication $\left(X_{1}^{\prime} X_{1}+k I\right)^{-1}\left(X_{1}^{\prime} X_{1}+d I\right)\left(X_{1}^{\prime} X_{1}\right)^{-1}$ in (28); we have

$$
\left(X_{1}^{\prime} X_{1}+k I\right)^{-1}\left(X_{1}^{\prime} X_{1}+d I\right) \beta_{1}^{* *}(V)=\widetilde{\beta}_{1}(k, d) .
$$

Then

$$
\widetilde{\beta}_{1}(k, d)=\left(X_{1}^{\prime} X_{1}+k I\right)^{-1}\left(X_{1}^{\prime} X_{1}+d I\right)(I, 0) \beta^{\prime *}(V) .
$$

Let $L=\left(X_{1}^{\prime} X_{1}+k I\right)^{-1}\left(X_{1}^{\prime} X_{1}+d I\right)(I, 0)$ and $S=(I, 0)$; by Lemma 4 , if we want to prove that $\widetilde{\beta}_{1}(k, d)$ is admissible estimator of $\beta_{1}$, we only need to prove $L T^{-1} L^{\prime} \leq L T^{-1} S^{\prime}$. Since $X_{1}^{\prime} X_{2}=0$, we may conclude that

$$
T=X^{\prime} V^{-1} X=\left(\sigma_{11} \sigma_{22}-\sigma_{12}^{2}\right)^{-1}\left(\begin{array}{cc}
\sigma_{22} X_{1}^{\prime} X_{1} & 0 \\
0 & \sigma_{11} X_{2}^{\prime} X_{2}
\end{array}\right) \text {. }
$$


Then

$$
\begin{aligned}
L T^{-1} L^{\prime}= & \left(\sigma_{11} \sigma_{22}-\sigma_{12}^{2}\right)\left(X_{1}^{\prime} X_{1}+k I\right)^{-1} \\
& \times\left(X_{1}^{\prime} X_{1}+d I\right)(I, 0)\left(\begin{array}{cc}
\sigma_{22} X_{1}^{\prime} X_{1} & 0 \\
0 & \sigma_{11} X_{2}^{\prime} X_{2}
\end{array}\right)^{-1} \\
& \times(I, 0)\left(X_{1}^{\prime} X_{1}+d I\right)\left(X_{1}^{\prime} X_{1}+k I\right)^{-1} \\
= & \frac{\sigma_{11} \sigma_{22}-\sigma_{12}^{2}}{\sigma_{22}}\left(X_{1}^{\prime} X_{1}+k I\right)^{-1}\left(X_{1}^{\prime} X_{1}+d I\right) \\
& \times\left(X_{1}^{\prime} X_{1}\right)^{-1}\left(X_{1}^{\prime} X_{1}+d I\right)\left(X_{1}^{\prime} X_{1}+k I\right)^{-1}, \\
L T^{-1} S^{\prime}= & \left(\sigma_{11} \sigma_{22}-\sigma_{12}^{2}\right)\left(X_{1}^{\prime} X_{1}+k I\right)^{-1}\left(X_{1}^{\prime} X_{1}+d I\right)(I, 0) \\
& \times\left(\begin{array}{c}
\sigma_{22} X_{1}^{\prime} X_{1} \\
0
\end{array} \sigma_{11} X_{2}^{\prime} X_{2}\right)^{-1}(I, 0) \\
= & \frac{\sigma_{11} \sigma_{22}-\sigma_{12}^{2}}{\sigma_{22}}\left(X_{1}^{\prime} X_{1}+k I\right)^{-1} \\
& \times\left(X_{1}^{\prime} X_{1}+d I\right)\left(X_{1}^{\prime} X_{1}\right)^{-1} .
\end{aligned}
$$

It is obvious that $L^{\prime} T^{-1} L^{\prime} \leq L^{\prime} T^{-1} S^{\prime}$. The proof is completed.

Theorem 6. For the SUR model (1), when $V$ is known, if $P_{1} P_{2}=$ $P_{2} P_{1}$, then $\widetilde{\beta}_{1}(k, d)$ is admissible estimator of $\beta_{1}$ in the class of linear estimators $\Phi$.

Proof. For SUR model (1), when $P_{1} P_{2}=P_{2} P_{1}$, by Lin [14], we obtain $\widetilde{\beta}_{1}=\beta_{1}^{*}(V)$; thus we obtain

$$
\begin{aligned}
\tilde{\beta}_{1}(k, d) & =\left(X_{1}^{\prime} X_{1}+k I\right)^{-1}\left(X_{1}^{\prime} X_{1}+d I\right) \tilde{\beta}_{1} \\
& =\left(X_{1}^{\prime} X_{1}+k I\right)^{-1}\left(X_{1}^{\prime} X_{1}+d I\right) \beta_{1}^{*}(V) \\
& =\left(X_{1}^{\prime} X_{1}+k I\right)^{-1}\left(X_{1}^{\prime} X_{1}+d I\right)(I, 0) \beta^{*}(V) \\
& =L \beta^{*}(V) .
\end{aligned}
$$

Similar to Theorem 5 , we only need to prove $L T^{-1} L^{\prime} \leq$ $L T^{-1} S^{\prime} ; L$ and $S$ are defined as Theorem 5:

$$
T=X^{\prime} V^{-1} X=\left(\sigma_{11} \sigma_{22}-\sigma_{12}^{2}\right)^{-1}\left(\begin{array}{cc}
\sigma_{22} X_{1}^{\prime} X_{1} & -\sigma_{21} X_{1}^{\prime} X_{2} \\
-\sigma_{12} X_{2}^{\prime} X_{1} & \sigma_{11} X_{2}^{\prime} X_{2}
\end{array}\right)
$$

Denote $T^{-1}=\left(\begin{array}{cc}T^{11} & T^{12} \\ T^{21} & T^{22}\end{array}\right)$; by Rao et al. [15], we have

$$
\begin{aligned}
T^{11}= & \frac{\sigma_{11} \sigma_{22}-\sigma_{12}^{2}}{\sigma_{22}}\left[I-\rho_{12}^{2}\left(X_{1}^{\prime} X_{1}\right)^{-1} X_{1}^{\prime} P_{2} X_{1}\right]^{-1} \\
& \times\left(X_{1}^{\prime} X_{1}\right)^{-1} .
\end{aligned}
$$

Then we have

$$
\begin{aligned}
L T^{-1} L^{\prime}= & \left(X_{1}^{\prime} X_{1}+k I\right)^{-1}\left(X_{1}^{\prime} X_{1}+d I\right) T^{11} \\
& \times\left(X_{1}^{\prime} X_{1}+d I\right)\left(X_{1}^{\prime} X_{1}+k I\right)^{-1} \\
= & \frac{\sigma_{11} \sigma_{22}-\sigma_{12}^{2}}{\sigma_{22}}\left(X_{1}^{\prime} X_{1}+k I\right)^{-1}\left(X_{1}^{\prime} X_{1}+d I\right) \\
& \times\left[I-\rho_{12}^{2}\left(X_{1}^{\prime} X_{1}\right)^{-1} X_{1}^{\prime} P_{2} X_{1}\right]^{-1}\left(X_{1}^{\prime} X_{1}\right)^{-1} \\
& \times\left(X_{1}^{\prime} X_{1}+d I\right)\left(X_{1}^{\prime} X_{1}+k I\right)^{-1}, \\
L T^{-1} S^{\prime}= & \left(X_{1}^{\prime} X_{1}+k I\right)^{-1}\left(X_{1}^{\prime} X_{1}+d I\right) T^{11} \\
= & \frac{\sigma_{11} \sigma_{22}-\sigma_{12}^{2}}{\sigma_{22}}\left(X_{1}^{\prime} X_{1}+k I\right)^{-1}\left(X_{1}^{\prime} X_{1}+d I\right) \\
& \times\left[I-\rho_{12}^{2}\left(X_{1}^{\prime} X_{1}\right)^{-1} X_{1}^{\prime} P_{2} X_{1}\right]^{-1}\left(X_{1}^{\prime} X_{1}\right)^{-1} .
\end{aligned}
$$

Obviously $L^{\prime} T^{-1} L^{\prime} \leq L^{\prime} T^{-1} S^{\prime}$. The proof is completed.

\section{Conclusion Remarks}

In this paper we consider the parameter estimation in seemingly unrelated regression system. A Liu-type estimator is proposed to overcome the multicollinearity in seemingly unrelated regression system. The superiority of the new estimator has been also discussed and the admissibility of the new estimator is also discussed.

\section{Conflict of Interests}

The author declares that there is no conflict of interests regarding the publication of this paper.

\section{Acknowledgments}

The author is grateful to the editor and the anonymous referee for the valuable comments which improved the quality of the paper. This work was supported by the Scientific Research Foundation of Chongqing University of Arts and Sciences (Grant no. R2013SC12), Program for Innovation Team Building at Institutions of Higher Education in Chongqing (Grant no. KJTD201321), and the National Natural Science Foundation of China (nos. 11201505, 71271227).

\section{References}

[1] A. Zellner, "An efficient method of estimating seemingly unrelated regressions and tests for aggregation bias," Journal of the American Statistical Association, vol. 57, no. 298, pp. 348-368, 1962.

[2] A. Zellner, "Estimators for seemingly unrelated equations: some exact finite sample results," Journal of the American Statistical Association, vol. 11, no. 58, pp. 977-992, 1963. 
[3] L. Wang, H. Lian, and R. S. Singh, "On efficient estimators of two seemingly unrelated regressions," Statistics \& Probability Letters, vol. 81, no. 5, pp. 563-570, 2011.

[4] M. Roozbeh, M. Arashi, and H. Niroumand, "On seemingly unrelated semi-parametric models," in Proceedings of the 58th World Statistical Congress, pp. 5125-5131, International Statistical Institute, 2011.

[5] S. R. Singh, L. C. Wang, and H. M. Song, "The superiorities of minimum bayes risk linear unbiased estimator in two seemingly unrelated regressions," Journal of Statistical and Econometric Methods, vol. 12, no. 3, pp. 153-174, 2013.

[6] N. S. Revankar, "Some finite sample results in the context of two seemingly unrelated regression equations," Journal of the American Statistical Association, vol. 69, no. 3, pp. 187-190, 1974.

[7] V. K. Srivastava and D. E. Giles, A Seemingly Unrelated Regression Equations Models, vol. 4, Marcel Dekker Inc., New York, NY, USA, 1987.

[8] S. G. Wang, "Covariance improvement estimate of the parameters in seemingly unrelated regression models," in Proceedings of the 2nd Japan China Symposium on Statistics, pp. 318-321, 1986.

[9] A. Y. Liu and S. G. Wang, "A biased estimator in two seemingly unrelated regression model," Chinese Journal of Applied Probability and Statistics, vol. 7, no. 3, pp. 266-274, 1991.

[10] A. Y. Liu, "A improved principal component regression estimator in two seemingly unrelated regression model," Application of Statistics and Management, vol. 12, no. 2, pp. 70-75, 1993.

[11] S. M. Qiu, Improved c-k Estimators of Parameters in Seemingly Unrelated Regression System, Hunan University, 2008.

[12] M. Roozbeh, M. Arashi, and M. Gasparini, "Seemingly unrelated ridge regression in semiparametric models," Communications in Statistics, Theory and Methods, vol. 41, no. 8, pp. 13641386, 2012.

[13] X. R. Chen, The Parameter Estimation in Linear Regression Model, Science Press, 1985.

[14] C. T. Lin, "A class of two-stage regression estimation system," Chinese Science Bulletin, vol. 15, no. 14, pp. 840-842, 1984.

[15] C. R. Rao, H. Toutenburg, Shalabh, and C. Heumann, Linear Models and Generalizations, Springer, Berlin, Germany, 2008. 


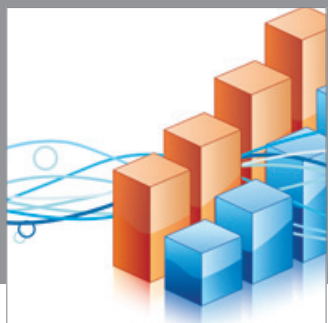

Advances in

Operations Research

mansans

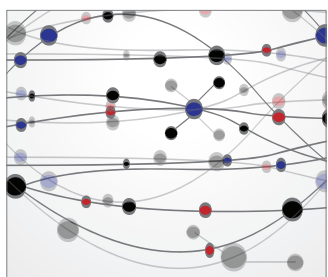

The Scientific World Journal
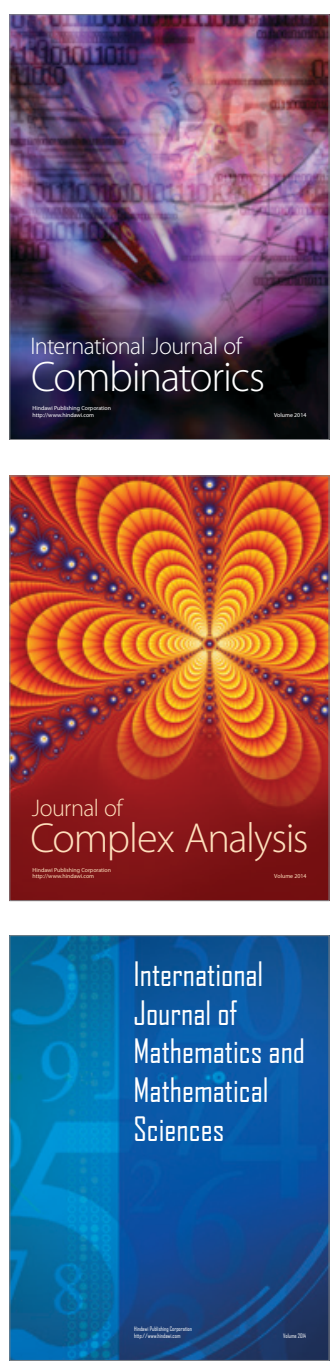
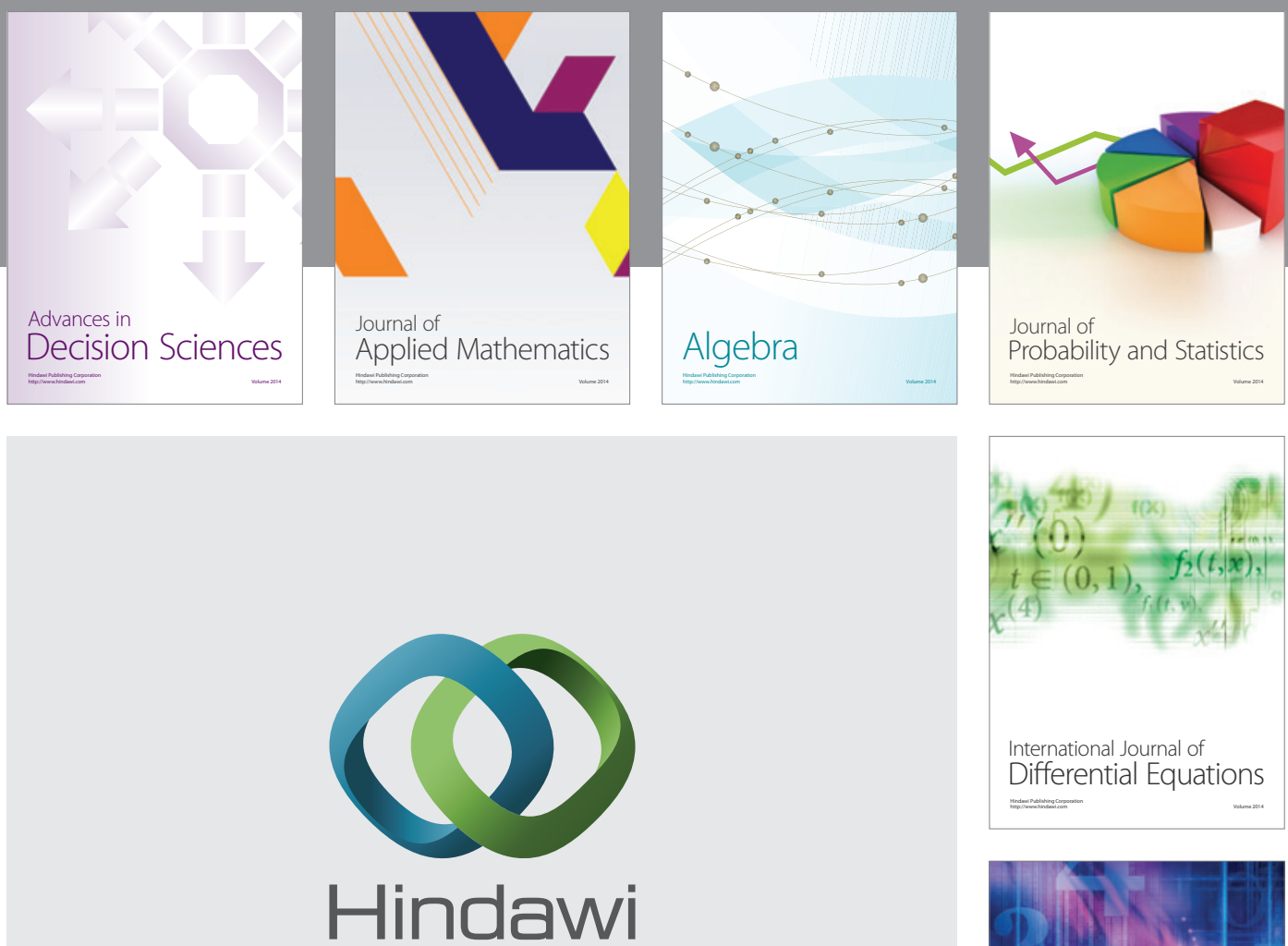

Submit your manuscripts at http://www.hindawi.com
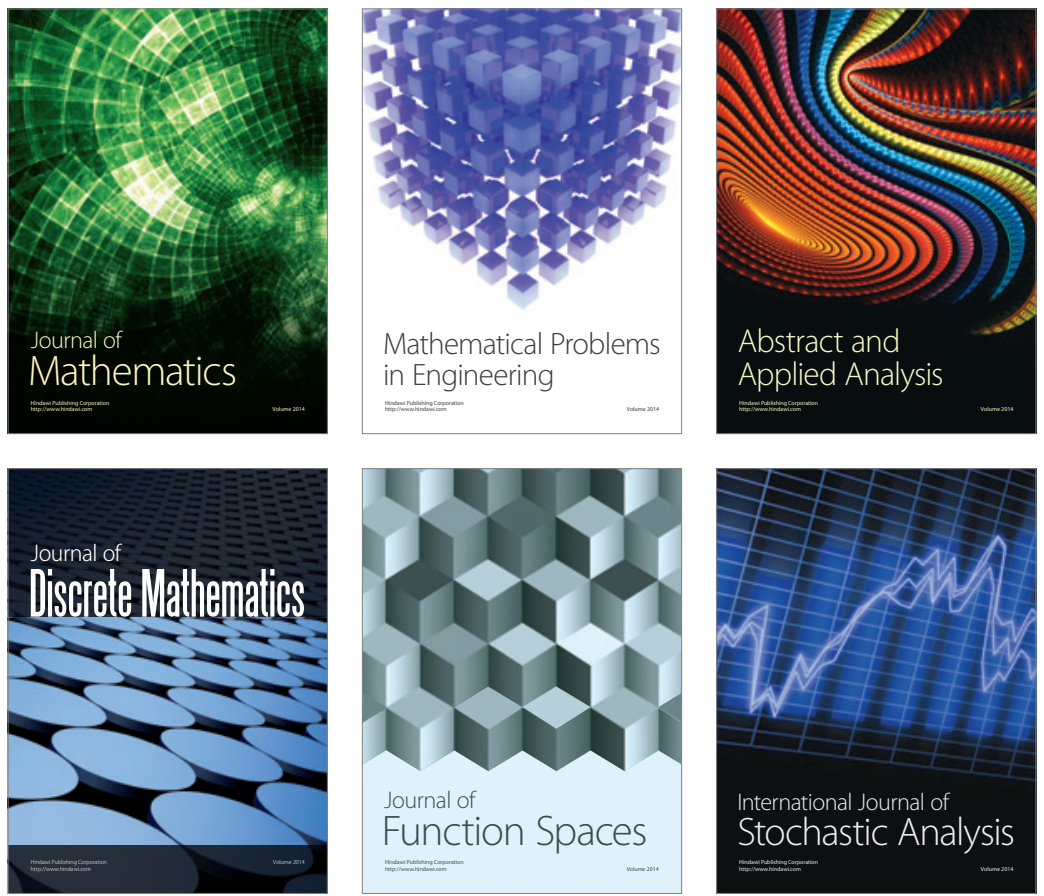

Journal of

Function Spaces

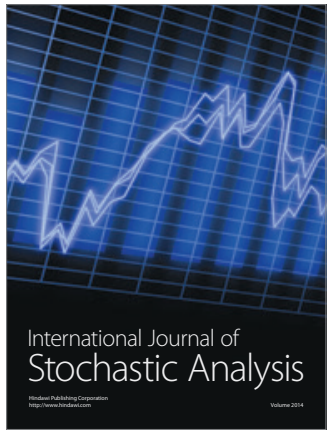

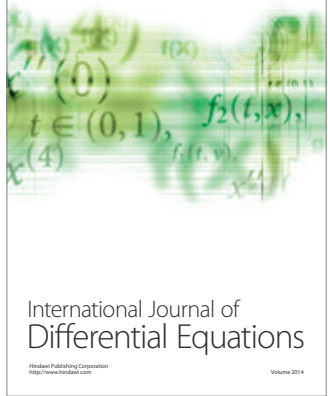
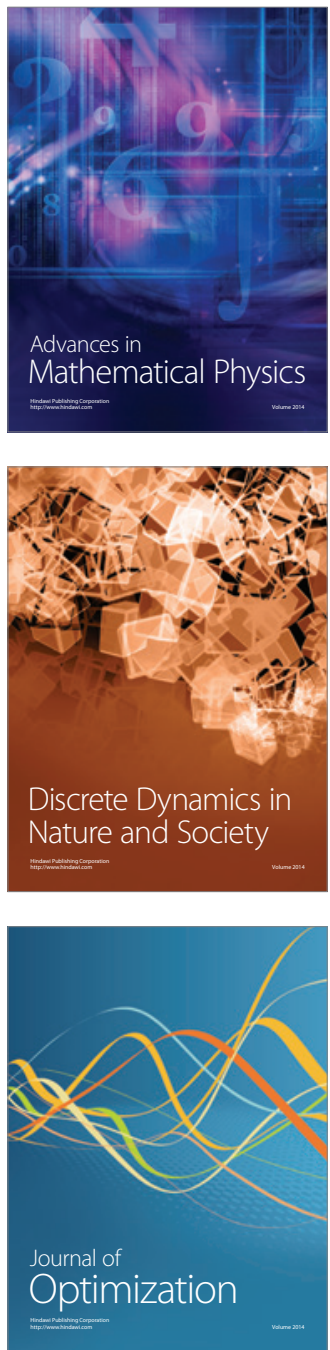